\title{
Impact of Short-Circuit Impedance Model for Variable-Speed Wind Turbine Generators on Operation of Overcurrent Relays
}

\author{
L. Somi, B. Polajžer and G. Štumberger \\ University of Maribor \\ Faculty of Electrical Engineering and Computer Science \\ Koroška cesta 46, SI-2000 Maribor \\ e-mail: bostjan.polajzer@um.si
}

\begin{abstract}
This paper discussed different short-circuit impedance models for type 3 and type 4 wind turbine generators. IEC and IEEE-PES models are either simplified or they require additional data. The proposed model is given by a non-linear dependency of the short-circuit impedance and voltage on the medium voltage side of the unit transformer. Furthermore, the proposed model, as well as the constant impedance-based model were used for timing coordination of overcurrent relays in a radial 6-bus network. The obtained results show that the constant impedance-based model overestimates short-circuit currents. Consequently, selectivity of individual overcurrent relays might be questionable.
\end{abstract}

Key words Overcurrent relay, short-circuit calculation, wind turbine generators.

\section{Introduction}

Current contributions from wind turbine generators (WTGs) during the short-circuits are considerably different than from conventional generators [1]-[3]. Main share of WTGs are variable-speed WTGs, especially type 3 and type 4 . Therefore, these two types have been widely analysed in the literature [4], [5]. WTGs, as well as other distributed generation units, might affect operation of overcurrent relays (OCRs). Constant short-circuit impedance model of generation units is typically used [6]-[9], which may not be consistent.

This paper is organized as follows. Section 2 describes IEC models [1] and IEEE-PES models [2],[3] of WTGs for calculation of short-circuit currents. While the IEC models are simplified, the IEEE-PES models require a set of characteristics. Therefore, a uniform model is proposed, containing a non-linear dependency of the short circuit impedance and the voltage on the medium voltage (MV) side of the WTG unit transformer. Timing coordination of OCRs operation is discussed in section 3. Results are given in section 4 for a fundamental radial 6-bus network where two WTGs models are applied, i.e., the constant impedance model and the proposed model. Finally, section 5 summarizes important conclusions and outlines future work.

\section{Short-Circuit Impedance Models for Variable-Speed Wind Turbine Generators}

Two types of variable-speed wind turbine generators are discussed, i.e., type 3 and type 4 . Type 3 is a doubly-fed asynchronous generator, where the stator is directly connected to the network, whilst the rotor is fed by an AC-DC-AC converter. The converter is typically rated for approximately $30 \%$ of the wind turbine's rated output power. Type 4 is a generator that is connected to the network through a full-size AC-DC-AC converter. The generator can be a synchronous machine, excited either by permanent magnets or by an excitation winding, or an asynchronous machine.

\section{A. IEC Models [1]}

New IEC standard [1] discusses different types of WTGs, where, for the calculation of short-circuit currents, WTGs and their unit transformers are combined into one unit. Short-circuit currents at the WTG's terminals are not dealt with this standard. Furthermore, in the cases when the WTG controls reactive power it is assumed that it acts as a controlled current source.

Type 3 WTGs are treated as constant impedance sources. The total positive-sequence impedance is given by

$$
Z_{\mathrm{WD}}=\sqrt{\frac{2}{3}} \frac{k_{\mathrm{WD}} U_{\mathrm{rTHV}}}{i_{\mathrm{WD} \max }}
$$

where $U_{\mathrm{rTHV}}$ is the rated voltage of the unit transformer at the high-voltage side, whilst $i_{\text {WDmax }}$ is the highest instantaneous value of the current in case of a three-phase short-circuit. The factor $k_{\mathrm{WD}}$ is for calculation of the peak short-circuit current and depends on the converter protection. If this factor is not known, then value of 1.7 is used. Furthermore, for a complex value $Z_{\mathrm{WD}}$ the ratio $R_{\mathrm{WD}} / X_{\mathrm{WD}}=0.1$ can be used, if it is not provided by the manufacturer. In the cases of unbalanced short-circuits the values given by the manufacturer shall be used. 


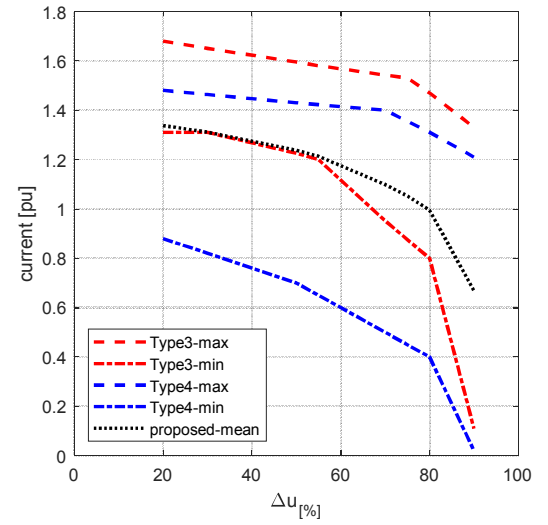

Fig. 1. Max, min and mean symmetrical short-circuit current magnitudes for 60-80 ms after fault application [2],[3].

Type 4 WTGs are treated as constant current sources. The source current depends on the type of the short-circuit, as well as on control strategy. Therefore, the value of the short-circuit current has to be provided by the manufacturer. Furthermore, WTG unit may be neglected if its contribution to a short-circuit current is not higher than $5 \%$. The same also applies to photovoltaic units.

\section{B. IEEE-PES Models [2],[3]}

The IEEE-PES model is based on a modified phasor approach [2],[3], where short-circuit currents are given as a function of the voltage on the MV side of the WTG unit transformer. In order to consider all valid operating conditions and control operating set-points, the shortcircuit characteristics are given by an envelope. Fig. 1. shows the short-circuit current envelopes for both WTGs types defined by the maximum and minimum currents, where $\Delta u_{[\%]}$ denotes a $\%$ of voltage at the MV bus. Furthermore, the current magnitudes shown in Fig. 1 are given for a time instant of 60-80 ms after fault application. Note, that magnitudes for a time instant immediately after the fault occurs are considerably higher. Moreover, for the unbalanced short-circuits, it is not possible to consider the positive and negative sequence components independently.

\section{Proposed Model}

IEC models of WTGs are simplified. However, they require manufacturers data. Furthermore, according to the IEEE-PES models WTGs cannot be treated as a constant impedance nor as constant current sources. A set of characteristics is required, which might not be available. Therefore, a WTG model is proposed as follows:

- Step 1: Mean current magnitudes are determined considering both WTG types and both envelopes, as shown in Fig. 1.

- $\quad$ Step 2: For the discussed interval $\Delta u_{[\%]} \in[20 \%, 90 \%]$ a per-unit values of the impedance are calculated using mean short-circuit current magnitudes.

- Step 3: The obtained dependency $z_{\mathrm{WTG}}\left(\Delta u_{[\%]}\right)$ in per unit is approximated with a 6 -th order polynomial (2).

$$
z_{\mathrm{WTG}[\mathrm{pu}]}=\sum_{i=0}^{6} a_{i}\left(\Delta u_{[\%]}\right)^{i}
$$

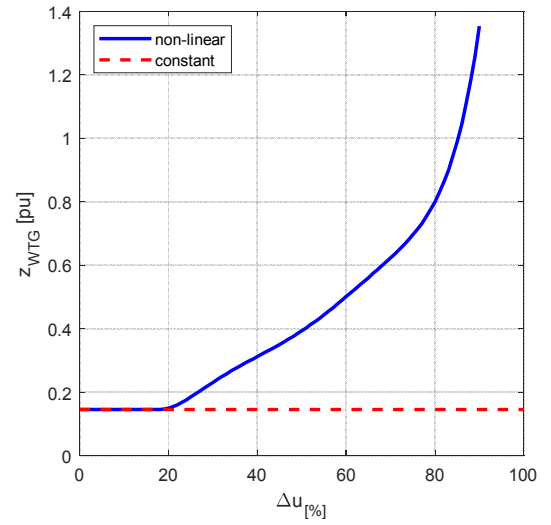

Fig. 2. Characteristics of the discussed WTG short-circuit impedance models.

Table I. Coefficients of the polynomial (2).

\begin{tabular}{ccccccc}
\hline$a_{0}$ & $a_{1}$ & $a_{2}$ & $a_{3}$ & $a_{4}$ & $a_{5}$ & $a_{6}$ \\
\hline 1.49 & -0.223 & $1.4 \cdot 10^{-2}$ & $-4.3 \cdot 10^{-4}$ & $7.1 \cdot 10^{-6}$ & $-6 \cdot 10^{-8}$ & $2 \cdot 10^{-10}$ \\
\hline
\end{tabular}

Coefficients of the polynomial $a_{i}$ are given in Table I. Fig. 2 shows the obtained non-linear short-circuit impedance model, where for values $\Delta u_{[\%]}<20 \%$ a constant impedance is assumed. Furthermore, it should be pointed out, that the proposed model is given only for the comparison with the constant impedance-based model (dashed line in Fig. 2), which is typically used for timing coordination of OCRs operation [6]-[9].

\section{Background for Parametrisation of OCRs Operation}

\section{A. Relay Notation and Relay Pairs}

Fig. 3 shows relay notation for a general case, where OCRs between the busses $B_{i}$ and $B_{j}$ are denoted as $R_{i j}$ and $R_{j i}$, whereas arrows denote the direction of OCRs operation. Thus, relays $R_{i j}$ and $R_{j i}$ will operate for a fault at location $\mathrm{FL}_{\mathrm{f}}$.

In order to attain timing coordination of OCRs operation, relay pairs are determined. An individual relay pair (RP) consists of a primary and a back-up OCR. Furthermore, each OCR might have one or more back-up OCRs. For a general case shown in Fig. 3, the back-up relays for the relay $R_{\mathrm{ij}}$ are marked in green colour, while back-up relays for the relay $R_{j i}$ are marked in blue colour. Note that $N_{i}$ and $\mathrm{N}_{\mathrm{j}}$ denote total number of lines connected to busses $B_{i}$ and $B_{j}$, respectively.

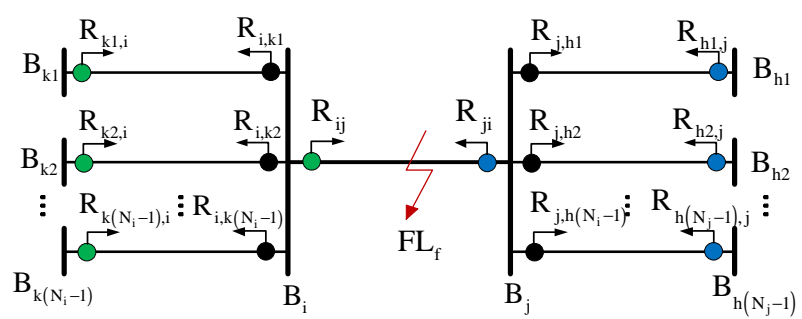

Fig. 3. Notation of OCRs and RPs. 


\section{B. Inverse-Time Characteristics}

Typically, OCRs with inverse-time characteristics are used in closed-loop and meshed networks. A general equation for an inverse-time characteristic is given by

$$
t_{\mathrm{ij}, \mathrm{f}}=T_{D \mathrm{ij}}\left(\frac{A}{\left(\frac{I_{\mathrm{ij}, \mathrm{f}}}{I_{P \mathrm{ij}}}\right)^{B}-1}+C\right)
$$

where $I_{\mathrm{ij}, \mathrm{f}}$ is the current passing through the relay $\mathrm{R}_{\mathrm{ij}}$ for a fault at location $\mathrm{FL}_{\mathrm{f}}$, whereas $I_{P \mathrm{ij}}$ and $T_{D \mathrm{ij}}$ denote the current pickup setting and the time dial setting, respectively. The constants $A, B$ and $C$ are given according to the characteristics type defined by the IEC [10] or IEEE [11] standards. They are summarized in Table II.

Table II. Constants of inverse-time characteristics.

\begin{tabular}{r|rcc|rcc}
\hline & \multicolumn{3}{|c}{ IEC } & \multicolumn{4}{c}{ IEEE } \\
Inverse type & $A$ & $B$ & $C$ & $A$ & $B$ & $C$ \\
\hline Standard & 0.14 & 0.02 & 0 & - & - & - \\
Long & 120.00 & 1.00 & 0 & - & - & - \\
Moderately & - & - & - & 0.05 & 0.02 & 0.114 \\
Very & 13.50 & 1.00 & 0 & 19.61 & 2.00 & 0.491 \\
Extremely & 80.00 & 2.00 & 0 & 28.20 & 2.00 & 0.122 \\
\hline
\end{tabular}

\section{Timing Coordination}

Selectivity is achieved by timing coordination, where the OCR intended to operate (primary relay) operates faster than other OCRs (back-up relays). Fig. 4 shows a fundamental RP, consisting of the primary relay $R_{i j}$ and the back-up relay $R_{k i}$. Selectivity for the $r$-th relay pair is assured when $\Delta t_{\mathrm{r}}>\mathrm{CTI}_{\mathrm{r}}$, where $\Delta t_{\mathrm{r}}$ is the difference of operating times of both relays for a fault at the location $\mathrm{FL}_{\mathrm{i}}$. Furthermore, $\mathrm{CTI}_{\mathrm{r}}$ is a coordination time interval which is determined according to specified time delays of both OCRs and operating times of both corresponding circuit breakers.

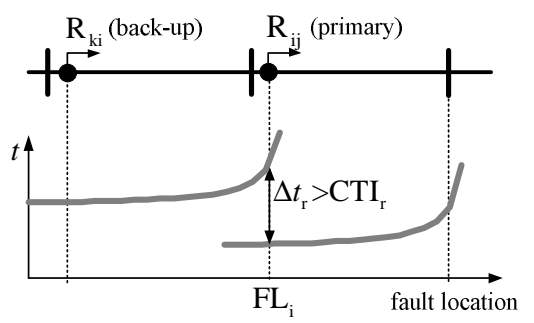

Fig. 4. Timing coordination for a fundamental RP.

Pickup current settings and time dial settings of all OCRs can be determined in different ways. In this paper an optimisation is proposed using differential evolution algorithm, which is a stochastic search algorithm [12]. The objective function is given by (4) as a sum of operating times of all primary and back-up relays,

$$
T_{\mathrm{opr}}=\sum_{\mathrm{r}=1}^{\mathrm{N}_{\mathrm{RP}}}\left(t_{[\mathrm{p}, \mathrm{r}]}+t_{[\mathrm{b}, \mathrm{r}]}\right)+p
$$

where $\mathrm{N}_{\mathrm{RP}}$ is the number of all RPs. The objective function (4) is minimized, which requires calculation of operating times of all primary and back-up relays. For the r-th RP, they are denoted by $t_{[\mathrm{p}, \mathrm{r}]}$ and $t_{[\mathrm{b}, \mathrm{r}]}$, respectively. They are calculated by (3), where $I_{\mathrm{ij}, \mathrm{f}}$ corresponds to the fault locations given by a primary OCR, e.g. $\mathrm{FL}_{\mathrm{i}}$ in Fig. 4. Furthermore, $p$ denotes penalties, which are applied when $\Delta t_{\mathrm{r}}<\mathrm{CTI}_{\mathrm{r}}$, or when violating limits of the pickup current and time dial settings.

\section{Pickup Current Limits}

In order to assure a reliable operation of relay $R_{i j}$ the pickup current should be set within the limits, which are given as

$$
\left(1-K \frac{e_{\mathrm{CT} \%}}{100}\right) I_{\mathrm{ij}, \min }>I_{P \mathrm{ij}}>\left(1+K \frac{e_{\mathrm{CT} \%}}{100}\right) I_{\mathrm{ij}, L \max }
$$

where $I_{\mathrm{ij}, \min }$ is a minimal short-circuit current passing through the discussed OCR, i.e., for a fault located at remote end of the discussed line. $I_{\mathrm{ij}, L \max }$ is a maximum value of the transient load current passing through the discussed OCR, whereas $e_{\mathrm{CT} \%}$ denotes the $\%$ error of current transformers. $K>1$ is a safety factor.

\section{Procedure for parametrisation of OCRs}

The following procedure was used:

- Step 1: Determine RPs and CTIs.

- Step 2: Calculate short-circuit currents for faults at all relay points, as well as at remote ends of all feeders.

- Step 3: Determine pickup current settings for all OCRs within given limits and the rule $I_{P \mathrm{ij}, \text { prim }}<I_{P_{\mathrm{ij}, \text { back }}}$.

- Step 4: For the chosen type of inverse-time characteristics determine the time dial settings for all OCRs, through minimisation of operating times.

\section{Results}

A $20 \mathrm{kV}$ radial 6-bus topology is discussed in [7], which is shown in Fig. 5. The source short-circuit power was set to 435 MVA, whilst an additional WTG with a rated power of 30 MVA was connected to the bus $B_{5}$. Table III shows RPs. Note, that line $\mathrm{L}_{56}$ is protected only by the relay $R_{56}$ since no source is assumed at $B_{6}$. Furthermore, $R_{12}$ and $R_{54}$ are not primary relays. However, they should operate before operation of the transformer protection relay at a source, and the WTG protection, respectively.

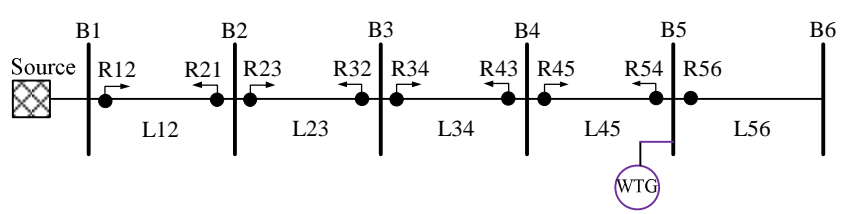

Fig. 5. Radial 6-bus network with WTG.

Table III. RPs for the radial 6-bus network (Fig. 5).

\begin{tabular}{r|ccccccc}
\hline RP no. & 1 & 2 & 3 & 4 & 5 & 6 & 7 \\
\hline primary & $\mathrm{R}_{23}$ & $\mathrm{R}_{34}$ & $\mathrm{R}_{45}$ & $\mathrm{R}_{56}$ & $\mathrm{R}_{21}$ & $\mathrm{R}_{32}$ & $\mathrm{R}_{43}$ \\
back-up & $\mathrm{R}_{12}$ & $\mathrm{R}_{23}$ & $\mathrm{R}_{34}$ & $\mathrm{R}_{45}$ & $\mathrm{R}_{32}$ & $\mathrm{R}_{43}$ & $\mathrm{R}_{54}$ \\
\hline
\end{tabular}




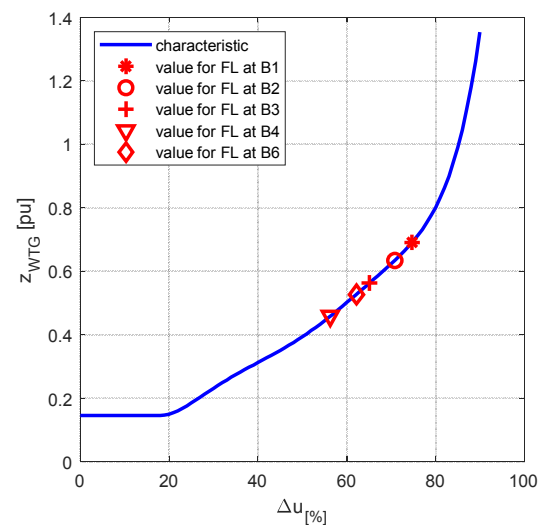

Fig. 6. Values of WTG's short-circuit impedance for different fault locations (FL).

\section{A. Short-Circuit Calculations}

Short-circuit calculations were performed using PSS $^{\circledR}$ Sincal Electricity Basic Package. WTG was incorporated as an impedance source. Two models were used, i.e., the proposed non-linear model and a constant impedance model, as shown in Fig. 2. The solution of the non-linear model has been achieved in iterations until the short-circuit current converged to a final value. A minimum of 9 iterations and a maximum of 15 iterations were needed. Values of the obtained WTG's short-circuit impedance for different fault locations are shown in Fig. 6. Note, that for the fault location at $\mathrm{B}_{5}$ the WTG was not considered.

Fig. 7 shows resulting short-circuit currents seen by individual relays for faults at remote line ends, e.g., for $\mathrm{R}_{12}$ the fault was simulated at $B_{2}$, whereas for $R_{21}$ the fault was simulated at $B_{1}$. The obtained results show that WTG model influences the short-circuit currents seen by OCRs that operate in the direction away from the WTG, i.e. $\mathrm{R}_{56}$ and $R_{21}$ to $R_{54}$. The short-circuit currents determined for the constant impedance WTG model are considerably higher. Consequently, the pickup current settings of the discussed relays could be higher when using constant WTG impedance. This might decrease OCRs sensitivity. Moreover, if the pickup settings were to high the OCRs might not pickup.
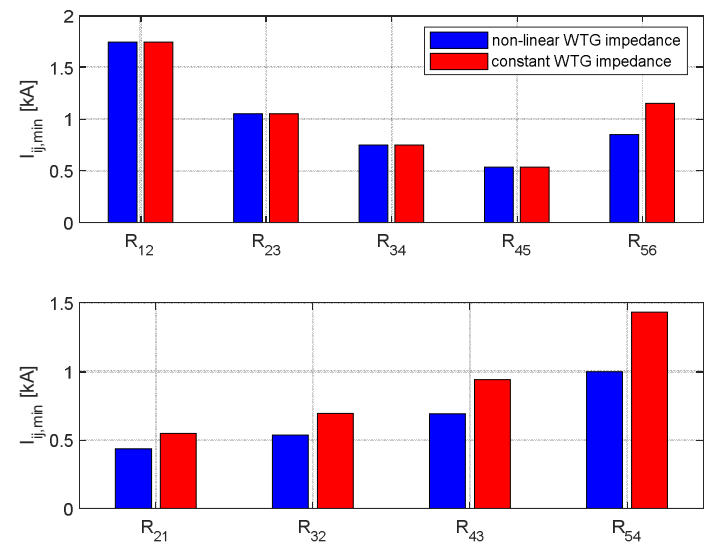

Fig. 7. Impact of WTG impedance on short-circuit currents seen by individual relays for remote faults.

\section{B. Settings of OCRs and Selectivity}

The current pickup settings were determined according to (5). The values shown in Fig. 7 were used for $I_{\mathrm{ij}, \mathrm{min}}$, whereas $I_{\mathrm{ij}, L \max }=250 \mathrm{~A}$ was used for all OCRs. Furthermore, $e_{\mathrm{CT} \%}=10 \%$ and $K=1.1$. In order to assure selectivity, the rule $I_{P \mathrm{ij}, \text { prim }}<I_{P \mathrm{ij}, \text { back }}$ was also applied.

The time dial settings were determined with the differential evolution (DE). The IEC very inverse-time characteristic and CTI $=200 \mathrm{~ms}$ were considered for all OCRs. Further objective was to decrease operating times of all primary OCRs below the value of $300 \mathrm{~ms}$. In this way the discussed OCRs operates faster than the transformer protection relay, which is typically set to $500 \mathrm{~ms}$ delay. The DE strategy rand/1/exp was used [12], where the step size and crossover probability constant were set as 0.8 and 0.5 , respectively. Optimization was finished after 220 iterations. The obtained time dial settings were rounded to $10 \mathrm{~ms}$, which is also the minimal possible setting. Two sets of time dial settings were calculated, i.e., one using a constant WTG impedance and one using a non-linear WTG impedance. Results are given in Table. IV.

\begin{tabular}{c|cc|cc}
\multicolumn{5}{c}{ Table IV. Two sets of OCRs settings. } \\
\hline \multirow{2}{*}{ OCR no. } & \multicolumn{2}{|c}{ non-linear } & $z_{\mathrm{WTG}}$ & \multicolumn{2}{c}{ constant } & $z_{\mathrm{WTG}}$ \\
& $I_{P \mathrm{ij}}[\mathrm{A}]$ & $\mathrm{T}_{D \mathrm{ij}}[\mathrm{ms}]$ & $I_{P \mathrm{ij}}[\mathrm{A}]$ & $\mathrm{T}_{D \mathrm{ii}}[\mathrm{ms}]$ \\
\hline $\mathrm{R}_{12}$ & 873 & 40 & 873 & 40 \\
$\mathrm{R}_{23}$ & 525 & 40 & 525 & 40 \\
$\mathrm{R}_{34}$ & 374 & 40 & 374 & 40 \\
$\mathrm{R}_{45}$ & 330 & 20 & 330 & 20 \\
$\mathrm{R}_{56}$ & 330 & 10 & 330 & 10 \\
$\mathrm{R}_{21}$ & 330 & 10 & 330 & 10 \\
$\mathrm{R}_{23}$ & 330 & 20 & 347 & 30 \\
$\mathrm{R}_{34}$ & 346 & 40 & 470 & 40 \\
$\mathrm{R}_{54}$ & 499 & 40 & 717 & 40 \\
\hline
\end{tabular}

Figs. 8-10 show $\Delta t_{\mathrm{r}}$ values of all RPs and operating times of all primary and backup OCRs. The CTI value of $200 \mathrm{~ms}$ is marked to check the selectivity, as well as the maximal operating time for primary OCRs, i.e. $300 \mathrm{~ms}$. Three cases were considered, as follows.

Case A: The OCRs setting, as well as $\Delta t_{\mathrm{r}}$ values and operating times were all determined using non-linear WTG impedance. The results are shown in Fig. 8. Selectivity is achieved, as well as required operating times of primary OCRs.

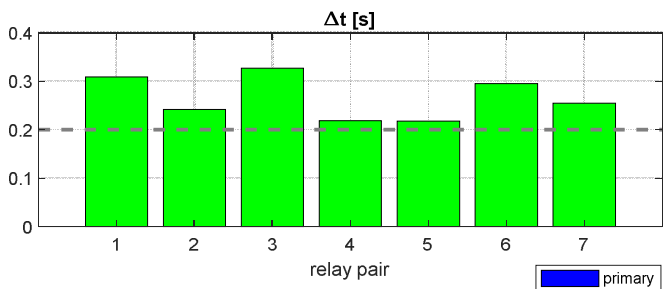
$t_{p}, t_{b}[s]$

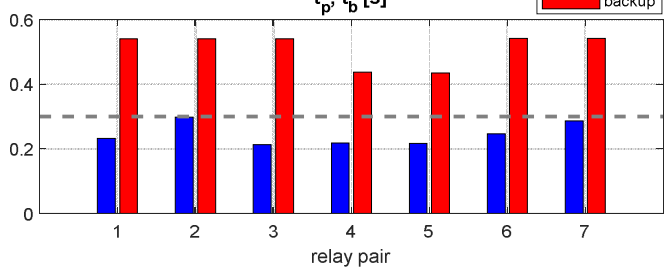

Fig. 8. $\Delta t_{\mathrm{r}}$ values and operating times - case A. 

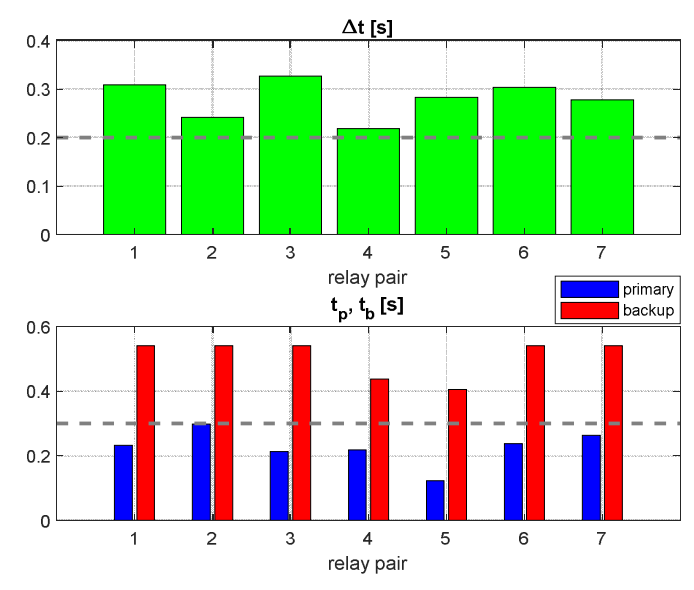

Fig. 9. $\Delta t_{\mathrm{r}}$ values and operating times - case B.

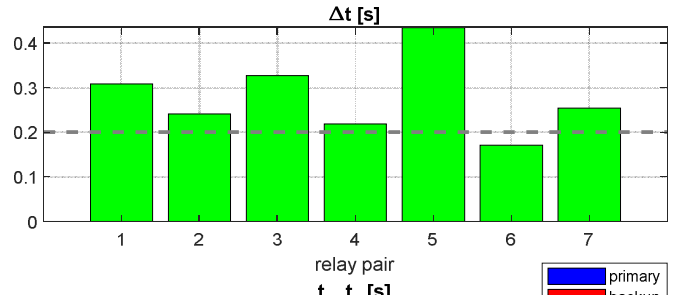

$t_{p}, t_{b}[s]$

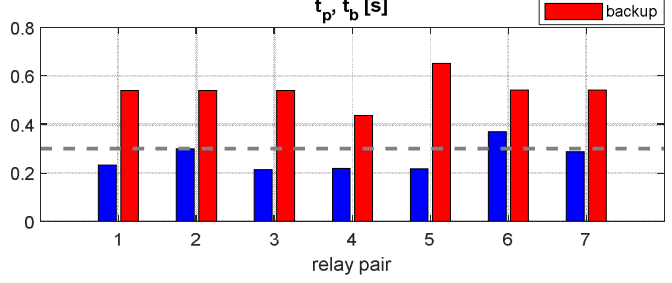

Fig. 10. $\Delta t_{\mathrm{r}}$ values and operating times - case C.
Case B: The OCRs setting, as well as $\Delta t_{\mathrm{r}}$ values and operating times were determined using constant WTG impedance. The results are shown in Fig. 9. Selectivity is achieved, as well as required operating times of primary OCRs.

Case C: The OCRs setting were determined using constant WTG impedance, which is typically used. However, $\Delta t_{\mathrm{r}}$ values and operating times were determined using nonlinear WTG impedance. The results are shown in Fig. 10. Selectivity is questionable, since $\Delta t_{6}<\mathrm{CTI}_{6}$. Furthermore, the operating time of $\mathrm{R}_{32}$ is higher than $300 \mathrm{~ms}$.

\section{Conclusion}

The proposed short-circuit impedance model for WTG is based on mean short-circuit current magnitudes, combining the type 3 and type 4 WTGs. Note that the proposed model is given only for the comparison with the constant impedance-based model, which is typically used for timing coordination of OCRs operation. Next, a fundamental 6-bus radial network topology was considered with an additional WTG. The timing coordination of OCRs was performed using the DE algorithm. The obtained results show that when using the constant impedance-based WTG model, the short-circuit currents were overestimated within the range between $26 \%$ and $44 \%$. Consequently, the operation of OCRs might be affected when using this model for timing coordination. It is shown that the sensitivity of OCRs might be reduced, whereas the selectivity might also be questionable. Further work should evaluate the impact of WTG models on OCRs operation for more complex topologies, like e.g. IEEE 14bus network.

\section{Acknowledgement}

This work has been supported by ARRS under Projects P2-0115 and L2-7556.

\section{References}

[1] IEC 60909-0 (2016): "Short-circuit currents in three-phase AC systems", Part 0: Calculation of currents, 2016.

[2] R. A. Walling, E. Gursoy, and B. English, "Current Contributions from Type 3 and Type 4 Wind Turbine Generators During Faults", IEEE PES T\&D Conference and Exposition, Orlando, FL, USA, May 7-10, 2012.

[3] IEEE PES Joint Working Group, "Fault Current Contributions from Wind Plants", 68th Annual Conference for Protective Relay Engineers 2015, TX, USA, March 3April 2, 2015.

[4] T. Kauffmann, U. Karaagac, I. Kocal, et. al. "Phasor Domain Modeling of Type III Wind Turbine Generator for Protection Studies", General Meeting of the IEEE PES, Denver, CO, USA, July 26-30, 2015.

[5] U. Karaagac, T. Kauffmann, I. Kocal, et. al. "Phasor Domain Modeling of Type IV Wind Turbine Generator for Protection Studies", General Meeting of the IEEE PES, Denver, CO, USA, July 26-30, 2015.

[6] H. H. Zeineldin, H. M. Sharaf, D. K. Ibrahim, and E. ElDin Abou El-Zahab, "Optimal Protection Coordination for Meshed Distribution Systems with DG Using Dual Setting Directional Over-Current Relays", IEEE Trans. Smart Grid, vol. 6, no. 1, pp. 115-123, 2015.

[7] V. C. Nikolaidis, E. Papanikolaou, and A. S. Safigianni, "A Communication-Assisted Overcurrent Protection Scheme for Radial Distribution Systems with Distributed Generation”, IEEE Trans. Smart Grid, vol. 7, no. 1, pp. 114-123, 2016.

[8] L. Huchel, and H.H. Zeineldin, "Planning the Coordination of Directional Overcurrent Relays for Distribution Systems Considering DG", IEEE Trans. Smart Grid, vol. 7, no. 3, pp. 1642-1649, 2016.

[9] K. Pereira, B. R. Pereira Jr., J. Contreras, and J. R. S. Mantovani, “A Multi-Objective Optimization Technique to Develop Protection Systems of Distribution Networks with Distributed Generation", IEEE Trans. Power Syst., vol. 33, no. 6, pp. 7064-7075, 2018.

[10] IEC 60255-151 (2016); "Measuring Relays and Protection Equipement", Part 151: Functional Requirements for Over/Under Current Protection”, 2009.

[11] IEEE Standard C37.112 (R2007); "Inverse-Time Characteristic Equations for Overcurrent Relays", 2007.

[12] K. V. Price, R. M. Storn, and J. A. Lampinen, Differential Evolution - A Practical Approach to Global Optimization, Springer, 2005. 